\title{
FATORES ASSOCIADOS À VIOLÊNCIA DOMÉSTICA NA GESTAÇÃO EM ADOLESCENTES E ADULTAS, EM FEIRA DE SANTANA, BAHIA.
}

\author{
$\underline{\text { Benvinda Matias Dantas Neta }}{ }^{1}$; Rosely Cabral de Carvalho ${ }^{2}$; Luciana Lima de \\ Matos $^{3}$ \\ 1. Bolsista PIBIC/CNPq, Graduando em Medicina, Universidade Estadual de Feira de Santana, e-mail: \\ benvinda.n1@gmail.com \\ 2. Orientadora, Departamento de Saúde, Universidade Estadual de Feira de Santana, e-mail: \\ roselycarvalho056@gmail.com \\ 3. Participante do NIEVS, Departamento de Saúde, Universidade Estadual de Feira de Santana, e-mail: \\ luluzinha_maia@hotmail.com
}

PALAVRAS-CHAVE: Violência doméstica; gestação; fatores associados

\section{INTRODUÇÃO}

A violência doméstica presente nas relações sociais entre homens e mulheres está profundamente arraigada às raízes históricas de assimetrias de poder, não sendo percebida como um problema grave e complexo, devido a invisibilidade de suas consequências, revelando as desigualdades socioculturais existentes entre os sexos de discriminação, abuso de poder (SCHRAIBER, 1999).

Nessa perspectiva, uma das formas dessa violência de gênero encontra-se presente em todos os estratos sociais. A violência contra a mulher como um fenômeno de caráter endêmico, multifatorial, revelando altas prevalências e se manifestando nos diversos meios sociais independentes de etnia, idade e condições socioeconômicas (BRASIL, 2011; AMARAL et al, 2013).

A violência doméstica pode atuar como modelo negativo nos ambientes familiares, gerando depressão durante a gestação e no pós-parto, partos prematuros, comportamentos prejudiciais à interação social e à saúde mental das crianças e adolescentes que convivem com estes modelos. Nessas famílias podem ocorrer relatos de ações que identificam negligência, violência física e psicológica, uso ou abuso de drogas e dificuldade de diálogo entre os membros. (HABIGZAND et al, 2005; AQUINO, 2009; SÁ et al, 2010; GAGNON et al, 2014; OKADA et al, 2015; ABDELHA et al, 2015; GEORGE et al, 2016; SILVA et al, 2016; RAO et al, 2016).

Na gestação pode ocorrer o aumento da frequência da violência contra a mulher e muitas vezes iniciar nesse período. A literatura destaca alguns fatores que associam violência doméstica com a gestação, como a baixa escolaridade, baixa renda familiar gravidez na adolescência, desemprego da gestante e do parceiro, consumo de álcool pelo parceiro, abuso sexual na infância, história prévia de violência doméstica e eventos estressantes de vida, multigestações, parceiros sem religiões, ter filhos de outros parceiros, história familiar de violência , idade mais elevada do parceiro (AUDI et al, 2008;SANTOS et al, 2010; SEMAHEGN et al, 2013; BAGCIOGLU et al, 2013; FAGEEH, 2014; SGOBERO et al, 2015 ; Lima et al, 2016 ).

As repercussões físicas, psicológicas e sociais dessa violência, assim como a instituição de medidas de superação pela vítima e sua família ganham relevância quando se escuta a trajetória percorrida e as interações sociais, vivenciadas pela mulher na sua aproximação com a violência. Torna-se necessário, portanto, ampliar a compreensão da violência durante o atendimento a esta mulher e sua família para melhor entender o fenômeno e viabilizar novas formas de intervenção, possibilitando boas práticas profissionais no enfrentamento e redução dos casos de violência contra mulheres.

Embora a violência contra as mulheres esteja incluída em agendas internacionais (saúde, direitos humanos, desenvolvimento e jurídico), ainda há uma lacuna entre o reconhecimento do problema e formas de melhor atendimento dessas mulheres em situação de violência. 


\section{MATERIAL E MÉTODOS (sujeitos/instrumentos/procedimentos)}

Foi realizada inicialmente uma revisão sistemática de estudos sobre a prevalência e fatores associados à violência doméstica na gestação nas bases de dados eletrônicas BVS; LILACS; SCIELO; MEDLINE (PUBMED), no período de 2005 a 2016. Os descritores utilizados foram: domestic violence; pregnancy; prevalence e factors associated. Os critérios de inclusão foram considerados artigos originais nacionais com ano de publicação 2005 a 2016 com delineamento transversal e publicados nos idiomas português, inglês e espanhol.

Concomitante a revisão sistemática foi realizada um estudo de corte transversal, no Hospital Inácia Pinto dos Santos (Hospital da Mulher), referência em urgências e emergências obstétricas, em Feira de Santana, Bahia.

Foram incluídas nesse estudo puérperas com 24 horas de pós-parto, atendidas na referida Instituição, tendo como critérios de exclusão mulheres no pós-parto imediato, óbitos fetais e as que tinham problemas psiquiátricos. O tamanho da amostra foi calculado a partir do total de 5.888 partos, no ano de 2015, com frequência esperada de $24,3 \%$ da violência doméstica na gestação. Erro amostral de 5\% e intervalo de confiança de 95\% chegando a um total de 324 mulheres (SILVANY NETO, 2008; FEIRA DE SANTANA, 2015).

Os dados foram coletados entre dezembro de 2017 a fevereiro de 2018, por meio de um questionário adaptado, aplicado em sala reservada, contendo variáveis socioeconômicas e demográficas, gineco-obstétricas, uso de drogas por parentes e relacionadas a violência. A variável dependente foi a presença de violência doméstica ou não e a presença da violência antes ou durante a gestação. As questões relacionadas à violência doméstica foram formuladas, utilizando-se como referência o questionário padronizado e validado no Brasil Abuse Assessment Screening (AAS) e adaptado e revalidado para a população em estudo, em maternidade de referência na cidade de Salvador, Bahia (SENA, 2014).

Os dados foram digitados duplamente com realização de validação no Epidata, armazenados, utilizando-se o pacote estatístico IBM SPSS, versão 20. Foi realizada uma análise descritiva, com distribuição das frequências para variáveis qualitativas, cálculo de média, mediana e desvio padrão para variáveis quantitativas. $\mathrm{Na}$ análise bivariada, foi aplicado teste Qui quadrado de Pearson, para identificar associações estatísticas entre as variáveis de estudo com um nível de significância de 5\% e IC 95\%.

A análise multivariada foi realizada no programa estatístico STATA, versão 10.0, através do modelo de regressão logística e com ajuste de variáveis que obtiveram o valor de $\mathrm{p}<0,05$, tendo como medida de efeito razão de chances (OR), com IC $95 \%$. Esta pesquisa tem aprovação do Comitê de Ética em Pesquisa da UEFS, sob CAAE 51273915.0.0000.0053 e número de protocolo 1.524.705.

\section{RESULTADOS E/OU DISCUSSÃO}

A amostra final dessa pesquisa foi composta por 324 puérperas com idade variando de 12 a 42 anos (média $=26,1$, mediana $=25$, desvio padrão $=6,7$ ). As adolescentes representaram a minoria (17,9\%). Declararam-se pretas e pardas 297 (92\%), a maioria eram casadas ou viviam em união estável 255 (78,7\%). Enquanto a escolaridade, em sua maior parte cursou ensino médio 191 (59\%) e eram dependentes financeiramente $254(78,4 \%)$. A renda familiar foi de até um salário mínimo em 164 $(50,6 \%)$ das entrevistadas. Em relação as variáveis obstétricas as maiores proporções de mulheres tiveram de 2 a 4 gestações $(48,6 \%)$ e mais de 2 partos $(63,2 \%)$. 
Entre o total da amostra $68(21 \%)$ mulheres afirmaram ter sofrido violência doméstica, 33 (48,5\%) antes da gestação e 35 (51,5\%) durante a gestação. Em relação aos tipos de violência, a psicológica esteve presente em sua maioria $(34,3 \%)$, moral $(23,8 \%)$, física $(19,3 \%)$, patrimonial $(11,6 \%)$ e sexual $(11 \%)$. O perpetrador da violência em sua maioria foi o ex- companheiro $(49,4 \%)$ seguido do companheiro $(26,5 \%)$, locais do corpo mais atingidos pelo agravo foram face $(39,3 \%)$ seguido de membros superiores $(19,7 \%)$, os problemas de saúde mais frequentes foram sintomas de cefaleias constantes e falta de apetite $(20,5 \%)$ e diminuição da libido $(16,9 \%)$ e a humilhação $(33,4 \%)$ foi o sentimento mais frequente das mulheres após a ocorrência da violência.

$\mathrm{Na}$ análise bivariada, as variáveis que apresentaram significância estatística foram uso de drogas pelo ex- companheiro $(\mathrm{p}=0,000)$ na tabela 2 e violência psicológica na forma de insulto, chantagem e ridicularização $(\mathrm{p}=0,010)$ na tabela 3.

Tabela 2 Associação de violência doméstica e uso de drogas por parentes de mulheres atendidas em uma maternidade de referência, em Feira de Santana, Bahia, 2016.

\begin{tabular}{|c|c|c|c|c|c|}
\hline \multirow[t]{3}{*}{ Uso de drogas $(n=324)$} & \multicolumn{4}{|c|}{ Presença da violência } & \multirow[t]{3}{*}{ Valor de $p$} \\
\hline & \multicolumn{2}{|c|}{ Sim } & \multicolumn{2}{|c|}{ Não } & \\
\hline & $\mathrm{n}$ & $\%$ & $\mathrm{~N}$ & $\%$ & \\
\hline Pai & 23 & 17,5 & 83 & 20,0 & 0,827 \\
\hline Mãe & 14 & 10,6 & 58 & 14,0 & 0,715 \\
\hline Companheiro & 34 & 25,8 & 125 & 30,2 & 0,864 \\
\hline Irmão & 18 & 13,6 & 60 & 14,5 & 0,603 \\
\hline Ex companheiro & 32 & 24,2 & 34 & 8,3 & $0,000 *$ \\
\hline Familiares & 11 & 8,3 & 54 & 13,0 & 0,368 \\
\hline
\end{tabular}

*Estatisticamente significante / Questões com respostas múltiplas

Tabela 3 Associação da presença da violência doméstica e tipos de violência psicológica em mulheres atendidas em uma maternidade de referência, em Feira de Santana, Bahia, 2016.

\begin{tabular}{lccccc}
\hline Formas da violência & \multicolumn{4}{c}{ Presença da violência } & Valor de p \\
& Antes da gestação & Durante a gestação & \\
& $\mathrm{n}$ & $\%$ & $\mathrm{~N}$ & $\%$ & \\
\hline Psicológica (n=189) & 28 & 33,3 & 34 & 35,0 & 0,074 \\
Insultou, chantageou, ridicularizou & 19 & 22,9 & 30 & 28,3 & $\mathbf{0 , 0 1 0}$ \\
Constrangeu & 20 & 24,1 & 26 & 24,6 & 0,228 \\
Ameaçou & 13 & 15,6 & 17 & 16,0 & 0,446
\end{tabular}




\begin{tabular}{lccccc} 
Controlou ações, comportamentos & 11 & 13,3 & 13 & 12,3 & 0,743 \\
Limitou direito de ir e vir & 11 & 13,3 & 05 & 4,7 & 0,064 \\
Vigiou e perseguiu & 06 & 7,2 & 10 & 9,4 & 0,313 \\
Aprisionou & 02 & 2,4 & 02 & 1,9 & 0,952 \\
Explorou & 01 & 1,2 & 03 & 2,8 & 0,332 \\
\hline
\end{tabular}

*Estatisticamente significante / Questões com respostas múltiplas

\section{CONCLUSÃO}

Diante dos resultados a violência doméstica se apresenta como prejudicial à saúde. Deve ser investigada nos serviços de pré-natal situações suspeitas ou relatos atentando para violência psicológica que se aponta em maiores proporções e qualquer intercorrência clínica que possa trazer danos à saúde materno-infantil.

Os resultados deste estudo devem ser interpretados com cautela, apesar da validação do instrumento utilizado e a calibração na coleta dos dados. Trata-se de um tema complexo e de difícil abordagem, por tratar-se de mulheres no período puerperal e pela exposição à violência das mulheres entrevistadas que podem ter omitido informações. Deve-se considerar também que estudos de prevalência não permitem inferências dos resultados para população geral. O presente estudo representa o perfil e os fatores associados de mulheres atendidas em uma maternidade pública de referência, em um município do interior da Bahia.

Dessa forma, entendem-se as políticas públicas como imprescindíveis na prevenção, combate e notificação dos casos de violência, com a integração, qualificação, adequação dos horários de funcionamento da rede de atendimento em benefício ao acesso nos serviços, enfrentamento do agravo e rompimento do ciclo, com enfoque na estimulação do autocuidado das vítimas para evitar consequências psicológicas.

\section{REFERÊNCIAS}

ABDELHAI, R.; MOSLEH, H. Screening for antepartum anxiety and depression and their association with domestic violence among Egyptian pregnant women. The Journal of the Egyptian Public Health Association, v. 90, n. 3, p. 101-108, 2015.

AMARAL, N. A.; AMARAL, C. A.; AMARAL, T.L. M. Mortalidade feminina e anos de vida perdidos por homicídio/agressão em capital brasileira após promulgação da Lei Maria da Penha. Texto \& Contexto Enfermagem, v. 22, n. 4, 2013.

AQUINO, N. M. R. et al. Violência sexual e associação com a percepção individual de saúde entre mulheres gestantes. Revista de Saúde Pública, v. 43, p. 954-960, 2009.

AUDI, C. A. F. et al. Violence against pregnant women: prevalence and associated factors. Rev. Saúde Pública, vol.42, n.5, p. 877-85. Epub July 31, 2008.

BAGCIOGLU, E. et al. Decrease in domestic violence during pregnancy: a study from Turkey. Journal of interpersonal violence, v. 29, n. 2, p. 203-216, 2014. 
BRASIL. Ministério da Saúde. Secretaria Nacional de Enfrentamento à Violência contra as Mulheres. Política Nacional de Enfrentamento à violência contra as mulheres. Secretaria de Políticas para as Mulheres - Presidência da República. Brasília, 2011.

FAGEEH, W. M.K. Factors associated with domestic violence: a cross-sectional survey among women in Jeddah, Saudi Arabia. BMJ open, v. 4, n. 2, p. e004242, 2014.

GAGNON, Anita J.; STEWART, D. E. Resilience in international migrant women following violence associated with pregnancy. Archives of women's mental health, v. 17, n. 4, p. 303-310, 2014.

GEORGE, C. et al. Antenatal depression in coastal South India: prevalence and risk factors in the community. International journal of social psychiatry, v. 62, n. 2, p. 141-147, 2016.

HABIGZANG, L. F. et al. Abuso sexual infantil e dinâmica familiar: aspectos observados em processos jurídicos. Psicologia: teoria e pesquisa. Brasília. Vol. 21, n. 3 (set./dez. 2005), p. 341-348, 2005.

LIMA, L. H. M. de; MATTAR, R.; ABRAHÃO, A. R. Domestic violence in pregnant women: a study conducted in the postpartum period of adolescents and adults. Journal of interpersonal violence, p. 0886260516650968, 2016.

VIDAL, L.M.S. Fatores associados a violência doméstica na gestação em adolescentes e adultas, em Feira de Santana, Bahia.2017.88f. Dissertação de mestrado em saúde coletiva, apresentada ao programa de Pós-graduação em Saúde Coletiva, Departamento de Saúde, Universidade Estadual de Feira de Santana, Feira de Santana, 2017.

OKADA, M. M. et al. Domestic violence against pregnant women. Acta Paulista de Enfermagem, v. 28, n. 3, p. 270-274, 2015.

RAO, D. et al. The impact of domestic violence and depressive symptoms on preterm birth in South India. Social psychiatry and psychiatric epidemiology, v. 51, n. 2, p. 225-232, 2016.

SÁ, D.G.F. et al. Fatores de risco para problemas de saúde mental na infância/adolescência. Psicologia: Teoria e Pesquisa, v. 26, n. 4, p. 643-652, 2010.

SANTOS, A.S.et al. Violência doméstica durante a gestação: um estudo descritivo em uma unidade básica de saúde no Rio de Janeiro. Cad. Saúde Colet. Rio de Janeiro, v.18, n. 4, p. 483-93, 2010.

SCHRAIBER, L. B.; D'OLIVEIRA, A. F. L. P. Violência contra mulheres: interfaces com a Saúde. Interface (Botucatu), v.3, n.5, p. 13-26, 1999.

SEMAHEGN, A.; BELACHEW, T.; ABDULAHI, M. Domestic violence and its predictors among married women in reproductive age in Fagitalekoma Woreda, Awi zone, Amhara regional state, North Western Ethiopia. Reproductive health, v. 10, n. 1, p. $63,2013$. 
SENA, C. D. DE. Fatores associados à violência doméstica em gestantes atendidas em uma maternidade pública, salvador. Dissertação de mestrado apresentada a Escola de Enfermagem, Programa de Pós-Graduação em Enfermagem Mestrado Acadêmico em Enfermagem, UFBA. Salvador, BA. 2014, 111p.

SILVA, M. M.J. et al. Depression in pregnancy. Prevalence and associated factors. Investigación y education en enfermeria, v. 34, n. 2, p. 342-350, 2016.

SILVANY-NETO, A. M. Bioestatística em segredos. Salvador. 2008. 203p.

SGOBERO, J. K.G.S. et al. Violência física por parceiro íntimo na gestação: prevalência e alguns fatores associados. Aquichan, v. 15, n. 3, p. 339-350, 2015. 